\title{
Evaluation of Lecturer Teaching Performance Using AHP and SAW Methods
}

\author{
Benny Daniawan \\ Buddhi Dharma University \\ Jl. Imam Bonjol No. 41 Karawaci Ilir, Tangerang, Indonesia \\ b3n2y.miracle@gmail.com
}

\begin{tabular}{|c|c|}
\hline Article history: & Abstract \\
\hline $\begin{array}{l}\text { Received } 3 \text { December 2018; } \\
\text { Revised } 4 \text { December 2018; } \\
\text { Accepted } 5 \text { December 2018; } \\
\text { Available online } 19 \text { December } 2018\end{array}$ & $\begin{array}{l}\text { The lecturer performance evaluation activity is the routine of an university in } \\
\text { continuously improving internal quality as an evaluation and development of } \\
\text { educational institutions. Buddhi Dharma University Tangerang, every semester }\end{array}$ \\
\hline $\begin{array}{l}\text { Evaluation of Lecturer } \\
\text { Teaching Performance } \\
\text { MCDM } \\
\text { AHP } \\
\text { SAW }\end{array}$ & $\begin{array}{l}\text { to the absence of an effective and efficient method in determining the results, } \\
\text { especially in the Faculty of Science and Technology, Information System } \\
\text { Departement. The assessment process is carried out by distributing questionnaire } \\
\text { papers and filled out by students. This study aims to analyze the results of the } \\
\text { questionnaire, calculated by combining the Analytical Hierarchy Process (AHP) } \\
\text { method for weighting and combined the Simple Addictive Weighting (SAW) } \\
\text { method for ranking. The results obtained were the level of criteria weighting } \\
\text { accuracy reached } 90.39 \% \text { with } 28 \text { lecturers which teaching } 47 \text { subjects in the } \\
\text { Information Systems Departement. }\end{array}$ \\
\hline
\end{tabular}

\section{INTRODUCTION}

Quality education and the increasing number of students at the Buddhi Dharma University in Tangerang, is a challenge for academics to provide more optimal services by facilitating students to achieve an optimal learning outcome. Lecturers as professional educators and scientists with the main task of transforming, developing, and disseminating Science, technology and art, through education, research, and dedication to community [1].

The activeness of lecturers and students is the main key to the success of teaching and learning process. A lecturer successful in the teaching and learning process, if the performance value obtained is good [2]. The quality of lecturers it's an important role in a university that wants to produce quality graduates [3].

Lecturers are considered good if they have good performance values and are reviewed from several aspects. For this reason, it is very necessary to do an analysis to determine the performance value of lecturers. The lecturer performance evaluation system is one of the benchmarks in knowing the results of the lecturers' performance while teaching in one semester. The performance results of each lecturer will be discussed in a management meeting to be assessed and taken action. The assessment process that is currently running uses a questionnaire filled in manually for assessment [4], and is distributed to each student when going to conduct the Final Semester Exam. The results of filling out the questionnaire, that will be collected by the officer and given to academic staff, staff will immediately input the results of the questionnaire into the system.

The management must be more careful in making decisions because the final results of questionnaire recap are used as a reference. For this reason, we need a calculation method that can measure the results of a questionnaire calculation based on the main criteria that have an influence on performance appraisal, while also being able to assist in decision making for management. One of the decision making methods that can be used is the AHP method [5]. This method is a framework for making decisions by arranging into a hierarchy so that we can weight the 10 assessment criteria in the questionnaire and use the SAW method to rank 28 lecturers teaching 47 subjects in the Information Systems Departement [6]. 


\section{METHODS}

Multi Criteria Decision Making (MCDM) provides strong decisions in domains where the best alternative selection is very complex. In MCDM decision making has been applied in many domains. This method helps choose the best alternative from many criteria that can be obtained by analyzing the scope of the criteria, weighting criteria, and choosing the optimal results using multi criteria decision making techniques [7].

Multi Criteria Decision Making relates to structure and solving decision problems, with involves many criteria to support decision making on the best solution. This is consistent with choosing the "best" alternative from an alternative set.

Analytical Hierarchy Process (AHP) method was developed by Thomas L. Saaty. AHP method is one of several methods that can be used in decision making systems by taking into account factors of perception, preference, experience and intuition. AHP combines personal judgments and values into one logical way. AHP can solve complex multicriteria problems into a hierarchy [8].

According to Saaty, hierarchy is defined as a representation of a complex problem in a multi-level structure where the first level is a goal, followed by a factor level, criteria, sub criteria, and the last level of the alternative. With hierarchy, a complex problem can be broken down into groups which are then organized into a hierarchical form, so that the problems will appear more structured and systematic.

Table I: Saaty Scale of Pairwise Comparisons [5]

\begin{tabular}{|c|l|l|}
\hline \multicolumn{3}{|c|}{ The Fundamental Scale for Pairwise Comparisons } \\
\hline $\begin{array}{c}\text { Intensity of } \\
\text { Importance }\end{array}$ & \multicolumn{1}{|c|}{ Definition } & \multicolumn{1}{c|}{ Explanation } \\
\hline 1 & Equal importance & $\begin{array}{l}\text { Two elements contribute equally to the } \\
\text { objective }\end{array}$ \\
\hline 3 & Moderate importance & $\begin{array}{l}\text { Experience and judgment moderately } \\
\text { favor one element over another }\end{array}$ \\
\hline 5 & Strong importance & $\begin{array}{l}\text { Experience and judgment strongly favor } \\
\text { one element over another }\end{array}$ \\
\hline 7 & Very strong importance & $\begin{array}{l}\text { One element is favored very strongly } \\
\text { over another, its dominance is } \\
\text { demonstrated in practice }\end{array}$ \\
\hline 9 & Extreme importance & $\begin{array}{l}\text { The evidence favoring one element } \\
\text { over another is of the highest possible } \\
\text { order of affirmation }\end{array}$ \\
\hline
\end{tabular}

Intensities of $2,4,6$, and 8 can be used to express intermediate values. Intensities of $1.1,1.2,1.3$, etc. can be used for elements that are very close in importance.

\section{Steps for AHP method are as follows:}

Step 1: A represents $n \times n$ Matrix pairwise comparison

$$
A=\left[\begin{array}{cccc}
1 & a_{12} & \ldots & a_{1 n} \\
a_{21} & 1 & \ldots & a_{2 n} \\
\ldots & \ldots & \ldots & \ldots \\
a_{n 1} & a_{n 2} & \ldots & 1
\end{array}\right]
$$

Step 2: Normalize the raw score by Geometric mean as given below:

$$
\begin{aligned}
& \mathrm{r}_{\mathrm{ij}}=\sqrt[j]{(\boldsymbol{a i 1})(\boldsymbol{a i 2}) \ldots \ldots(\boldsymbol{a i j})} \quad \mathrm{i}, \mathrm{j}=1,2, \ldots \mathrm{n} \\
& \mathrm{r}=\left(\begin{array}{c}
r 1 \\
r 2 \\
\cdot \\
\cdot \\
r n
\end{array}\right)
\end{aligned}
$$


Step 3: Calculate Priority Vector or Eigen Value dan Row Matrix

P.V $\quad=r_{i j} / \Sigma r_{i j}$

Row Matrix $=\sum_{j=1}^{n} \boldsymbol{a i j} * \boldsymbol{P V} \mathbf{j 1}$

Step 4: Divided all the elements of the Row Matrix by their respective Priority Vector element to get Consistency Vector.

C.V = Row Matrix / P.V

Step 5: To avoid inconsistency in the pair wise comparison matrix, Saaty suggested the use of the maximum Priority Vector or Eigen Value ג max to calculate the effectiveness of judgement. The maximum Priority Vector or Eigen Value ג max can be determined as follows:

$\lambda \max =\Sigma \mathrm{C} . \mathrm{V} / \mathrm{n}$

Step 6: Estimate Index Consistency

C.I $=(\lambda \max -\mathrm{n}) /(\mathrm{n}-1)$

Step 7: Calculate Consistency Ratio (C.R) based on the R.I table. Shown in table 2.

C.R = C.I / R.I

For consistent value, it must: $0 \leq \mathrm{C} . \mathrm{R} \leq 0.1$

Table II: Random Index

\begin{tabular}{|c|c|c|c|c|c|c|c|c|}
\hline $\mathrm{n}$ & 1 & 2 & 3 & 4 & 5 & 6 & 7 & 8 \\
\hline R.I & 0 & 0 & 0.58 & 0.9 & 1.12 & 1.24 & 1.32 & 1.41 \\
\hline
\end{tabular}

\begin{tabular}{|c|c|c|c|c|c|c|c|}
\hline $\mathrm{n}$ & 9 & 10 & 11 & 12 & 13 & 14 & 15 \\
\hline R.I & 1.45 & 1.49 & 1.51 & 1.48 & 1,56 & 1,57 & 1,59 \\
\hline
\end{tabular}

Simple Additive Weighting (SAW) method is known as a combination of Linear Weighting or the simplest Scoring technique and is often used as one of the Multi Criteria Decision Making (MCDM). The scoring score is calculated based on each alternative by multiplying the value with the weight which determined by the Decision Maker.

\section{Steps for SAW Method are as follows:}

Step 1: Looking for the Benefit (+) and Cost (-) value from the criteria

Step 2: Construct a decision matrix $(\mathrm{m} \mathrm{x} \mathrm{n})$ includes $\mathrm{m}$ Alternative and $\mathrm{n}$ Criteria. Calculate the normalize decision matrix for benefit:

$\mathrm{r}_{\mathrm{ij}}=\frac{\mathrm{aij}}{\text { Max aij }} \quad \mathrm{a}=$ matrix value $\mathrm{i}=$ alternative and $\mathrm{j}=$ criteria where $\mathrm{i}, \mathrm{j}=1,2, \ldots \mathrm{n}$ 
Calculate the normalize decision matrix for cost:

$\mathrm{r}_{\mathrm{ij}}=\frac{\text { Min aij }}{\text { aij }} \quad \mathrm{a}=$ matrix value $\mathrm{i}=$ alternative and $\mathrm{j}=$ criteria where $\mathrm{i}, \mathrm{j}=1,2, \ldots \mathrm{n}$

Step 3: evaluate each alternative by Calculate Preference Value (Vi)

$\mathrm{Vi}=\sum_{j=1}^{n} \mathrm{Wj} *$ rij $\quad \mathrm{Wj}=$ Weighted Criteria from AHP and rij $=$ Normalize Decision Matrix

Where rij is the score of the $\mathrm{i}$ alternative, $\mathrm{Wj}$ is the weighted criteria [9]. With combine two types of methods, it's designed to select the best lecturers in University. The way of the data collection that is by questionnaire and using AHP Comparison Matrix the priority vector or weights of criteria will be computed. After computing weights of criteria, specifying of Consistency Rate will be executed. Consistency of the data is $0 \leq \mathrm{C} . \mathrm{R} \leq 0.1$, if more than 0.1 , the pairwise comparison needs revision. After the data it indicates sufficient consistency, we will use SAW method for ranking personnel.

\section{RESULTS AND DISCUSSION}

In this study the data used is obtained from student questionnaires. The data taken in the form of an assessment Index from 10 indicators of the teaching learning process that is [3]:

E1) Accuracy of time in teaching and effectiveness in a delivery content

E2) Clarity in a delivery of content and giving examples

E3) Motivate students and encourage participation in the classroom for discussion

E4) Openness in the assessment tasks and exams

E5) Openness in assisting the provision of information to learn

E6) The suitability content with the guidelines of teaching

E7) Utilize another tool like projectors etc

E8) The suitability of content with exam.

E9) Updates and the relevance of content under current conditions

E10) Overall assessment

The parameters of the questionnaire filled out by the students were:
A $($ Very Good $)=4$
$\mathrm{B}(\mathrm{Good})=3$
$\mathrm{C}($ Enough $)=2$
$\mathrm{D}(\mathrm{Bad}) \quad=1$

The accumulated value will show a Lecturer Achievement Index value. By using ten criteria as above, the university wants to rank twenty seven lecturers who have been assessed from fourty seven class and each class has ten to thirty five students. The weights or Priority Vector of criteria is calculated with Comparison Matrix [10], data was gathered from one expert opinion with questionnaire. It shown in Table III indicating the relation of criteria, and Table IV shown the calculate of Row Matrix.

Table III. Priority Vector of Criteria by Pairwise Comparison matrix

\begin{tabular}{|c|c|c|c|c|c|c|c|c|c|c|c|}
\hline Criteria & E1 & E2 & E3 & E4 & E5 & E6 & E7 & E8 & E9 & E10 & P.V \\
\hline E1 & 1 & 1 & 1 & 1 & 3 & 0.33333 & 3 & 0.33333 & 1 & 0.33333 & 0.0872 \\
\hline E2 & 1 & 1 & 1 & 1 & 1 & 1 & 3 & 3 & 1 & 1 & 0.1086 \\
\hline E3 & 1 & 1 & 1 & 1 & 1 & 0.33333 & 5 & 3 & 0.33333 & 1 & 0.0962 \\
\hline E4 & 1 & 1 & 1 & 1 & 1 & 0.33333 & 3 & 3 & 0.33333 & 1 & 0.0895 \\
\hline E5 & 0.33333 & 1 & 1 & 1 & 1 & 0.33333 & 3 & 3 & 0.33333 & 0.33333 & 0.0758 \\
\hline E6 & 3 & 1 & 3 & 3 & 3 & 1 & 3 & 1 & 1 & 1 & 0.1552 \\
\hline E7 & 0.33333 & 0.33333 & 0.2 & 0.33333 & 0.33333 & 0.33333 & 1 & 0.33333 & 0.33333 & 0.33333 & 0.0316 \\
\hline E8 & 3 & 0.33333 & 0.33333 & 0.33333 & 0.33333 & 1 & 3 & 1 & 1 & 0.33333 & 0.0801 \\
\hline E9 & 1 & 1 & 3 & 3 & 3 & 1 & 3 & 1 & 1 & 1 & 0.1416 \\
\hline E10 & 3 & 1 & 1 & 1 & 3 & 1 & 3 & 3 & 1 & 1 & 0.1342 \\
\hline Total & $\mathbf{1 4 . 6 6 6 7}$ & $\mathbf{8 . 6 6 6 7}$ & $\mathbf{1 2 . 5 3 3 3}$ & $\mathbf{1 2 . 6 6 6 7}$ & $\mathbf{1 6 . 6 6 6 7}$ & $\mathbf{6 . 6 6 6 7}$ & $\mathbf{3 0 . 0 0 0 0}$ & $\mathbf{1 8 . 6 6 6 7}$ & $\mathbf{7 . 3 3 3 3}$ & $\mathbf{7 . 3 3 3 3}$ & 1.0000 \\
\hline
\end{tabular}


Table IV. Calculate Row Matrix

\begin{tabular}{|c|c|c|c|c|c|c|c|c|c|c|c|c|}
\hline Criteria & E1 & E2 & E3 & E4 & E5 & E6 & E7 & E8 & E9 & E10 & P.V & Row Matrix \\
\hline E1 & 1 & 1 & 1 & 1 & 3 & 0.33333 & 3 & 0.33333 & 1 & 0.33333 & 0.0872 & 0.9684 \\
\hline E2 & 1 & 1 & 1 & 1 & 1 & 1 & 3 & 3 & 1 & 1 & 0.1086 & 1.2234 \\
\hline E3 & 1 & 1 & 1 & 1 & 1 & 0.33333 & 5 & 3 & 0.33333 & 1 & 0.0962 & 1.0886 \\
\hline E4 & 1 & 1 & 1 & 1 & 1 & 0.33333 & 3 & 3 & 0.33333 & 1 & 0.0895 & 1.0255 \\
\hline E5 & 0.33333 & 1 & 1 & 1 & 1 & 0.33333 & 3 & 3 & 0.33333 & 0.33333 & 0.0758 & 0.8779 \\
\hline E6 & 3 & 1 & 3 & 3 & 3 & 1 & 3 & 1 & 1 & 1 & 0.1552 & 1.7605 \\
\hline E7 & 0.33333 & 0.33333 & 0.2 & 0.33333 & 0.33333 & 0.33333 & 1 & 0.33333 & 0.33333 & 0.33333 & 0.0316 & 0.3416 \\
\hline E8 & 3 & 0.33333 & 0.33333 & 0.33333 & 0.33333 & 1 & 3 & 1 & 1 & 0.33333 & 0.0801 & 0.9013 \\
\hline E9 & 1 & 1 & 3 & 3 & 3 & 1 & 3 & 1 & 1 & 1 & 0.1416 & 1.5861 \\
\hline E10 & 3 & 1 & 1 & 1 & 3 & 1 & 3 & 3 & 1 & 1 & 0.1342 & 1.5495 \\
\hline
\end{tabular}

We will get Consistency Vector by divided Row Matrix with Priority Matrix. It shown in Table V.

Table V. Calculate Consistency Vector

\begin{tabular}{|c|}
\hline Row M atrix \\
\hline 0.9684 \\
\hline 1.2234 \\
\hline 1.0886 \\
\hline 1.0255 \\
\hline 0.8779 \\
\hline 1.7605 \\
\hline 0.3416 \\
\hline 0.9013 \\
\hline 1.5861 \\
\hline 1.5495 \\
\hline
\end{tabular} \mid \begin{tabular}{|c|}
\hline P.V \\
\hline 0.0872 \\
\hline 0.1086 \\
\hline 0.0962 \\
\hline 0.0895 \\
\hline 0.0758 \\
\hline 0.1552 \\
\hline 0.0316 \\
\hline 0.0801 \\
\hline 0.1416 \\
\hline 0.1342 \\
\hline \\
\hline \\
\hline \\
\hline \\
\hline \\
\hline \\
\hline \\
\hline 11.11 .2676 \\
\hline 11.4599 \\
\hline 11.5742 \\
\hline 11.3401 \\
\hline 11.8236 \\
\hline 11.2482 \\
\hline 11.5450 \\
\hline
\end{tabular}

$$
\begin{aligned}
& \lambda \operatorname{Max}=\frac{11.1060+11.2676+11.3217+11.4599+11.5742+11.3401+10.8236+11.2482+11.2005+11.5450}{10}=11.2887 \\
& \text { C.I }=\frac{11.2887-10}{10-1}=0.1432 \\
& \text { R.I }(10)=1.49 \\
& \text { C.R }=\frac{0.1432}{1.49}=0.0961 \text { (Consistent) }
\end{aligned}
$$

The Consistency Rate calculated was 0.0961 that is less than 0.1, indicating sufficient consistency [11]. Fig. 3 shows the developed hierarchical structure of the problem in which the first level has the goal of selecting the best lecturer performance evaluation in teaching learning. The second level consist of ten criteria. And the last level of the hierarchy comprises of the alternatives.

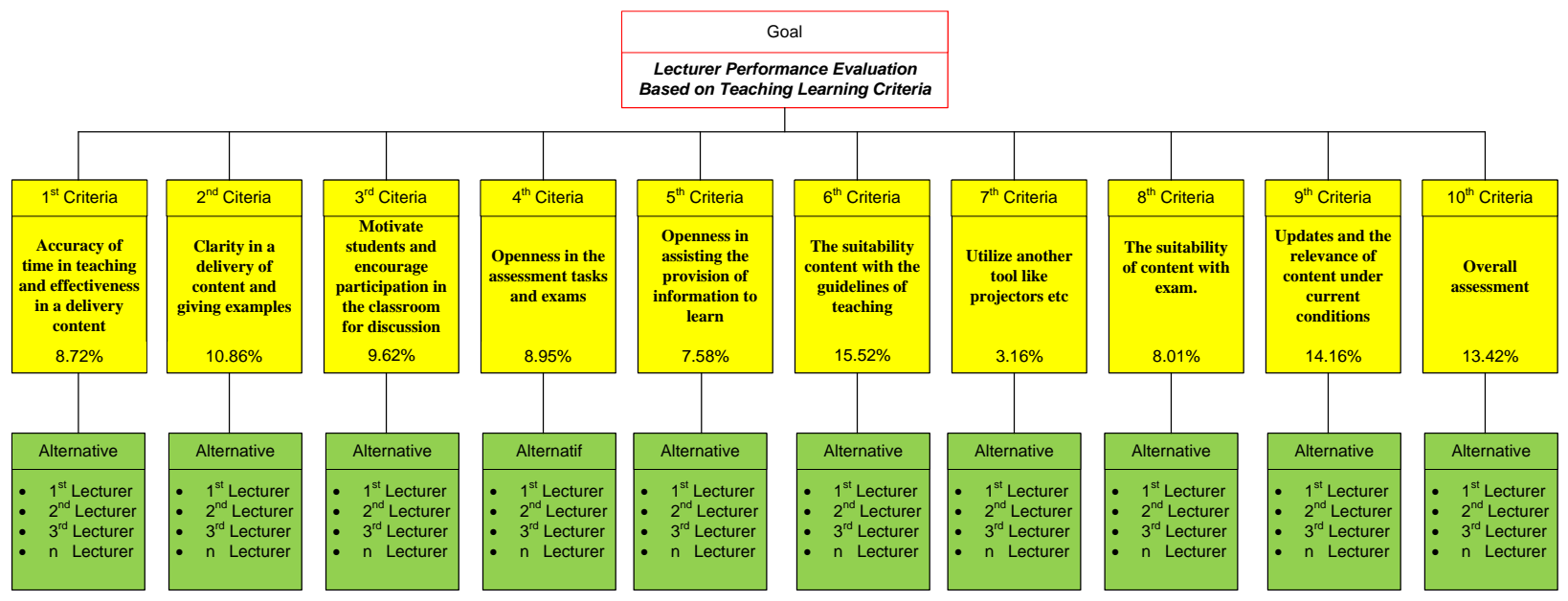

Fig. 3 Hierarchy structure of lecturer performance evaluation 
Based on scale values 1-4 from the students, we have calculate the average value from each alternative by initial, it shown in table VI. After computing priority vector of criteria in Table III, we will show procedure of SAW method:

Table VI. Collected Data based on scale 1-4

\begin{tabular}{|c|c|c|c|c|c|c|c|c|c|c|c|c|}
\hline \multirow[b]{2}{*}{ No } & \multirow[b]{2}{*}{ Courses } & \multirow{2}{*}{ Alternative } & \multicolumn{10}{|c|}{ Criteria } \\
\hline & & & E1 & E2 & E3 & E4 & E5 & E6 & E7 & E8 & E9 & E10 \\
\hline 1 & Database Management System & A.B & 3.21 & 2.86 & 2.86 & 3.14 & 3.12 & 3.00 & 3.14 & 3.14 & 2.86 & 3.00 \\
\hline 2 & Entrepreneurship and Motivation & A.L & 3.43 & 3.64 & 3.68 & 3.32 & 3.43 & 3.50 & 3.57 & 3.54 & 3.54 & 3.57 \\
\hline 3 & Database Management System & Alb & 3.29 & 2.81 & 3.06 & 2.97 & 2.97 & 2.97 & 3.06 & 2.90 & 2.94 & 2.97 \\
\hline 4 & Human Computer Interaction & A.H & 3.15 & 3.00 & 3.08 & 3.00 & 2.92 & 3.08 & 3.08 & 2.85 & 3.00 & 3.00 \\
\hline 5 & English Language 2 & A.P & 3.77 & 3.85 & 3.54 & 3.62 & 3.77 & 3.62 & 3.46 & 3.69 & 3.62 & 3.85 \\
\hline 6 & English Language 4 & A.P & 3.50 & 3.50 & 3.50 & 3.60 & 3.40 & 3.40 & 3.20 & 3.50 & 3.30 & 3.40 \\
\hline 7 & Strategic Information System & And & 2.77 & 2.88 & 3.00 & 2.92 & 2.88 & 2.92 & 2.96 & 2.96 & 3.04 & 2.92 \\
\hline 8 & E-Business & And & 3.11 & 3.11 & 2.89 & 2.89 & 3.00 & 3.22 & 3.22 & 3.11 & 3.00 & 3.11 \\
\hline 9 & TroubleShooting & Ams & 3.58 & 3.62 & 3.54 & 3.54 & 3.58 & 3.58 & 3.58 & 3.54 & 3.58 & 3.50 \\
\hline 10 & Mandarin Language & B.D & 3.12 & 3.00 & 2.84 & 3.08 & 3.00 & 3.16 & 3.24 & 3.16 & 3.04 & 3.12 \\
\hline 11 & Management Information Systems & D.W & 2.81 & 3.00 & 2.96 & 3.15 & 3.07 & 3.11 & 3.26 & 3.11 & 3.07 & 3.11 \\
\hline 12 & Entrepreneurship and Motivation & D.L & 3.25 & 3.19 & 3.56 & 2.88 & 3.25 & 3.31 & 3.44 & 3.13 & 3.13 & 3.38 \\
\hline 13 & Management & D.L & 3.53 & 3.40 & 3.47 & 3.47 & 3.20 & 3.40 & 3.33 & 3.33 & 3.47 & 3.40 \\
\hline 14 & Entrepreneurship and Motivation & D.L & 3.44 & 3.53 & 3.47 & 3.38 & 3.44 & 3.50 & 3.50 & 3.31 & 3.47 & 3.50 \\
\hline 15 & Object Oriented Programming & D.S & 3.56 & 3.44 & 3.50 & 3.44 & 3.56 & 3.50 & 3.63 & 3.38 & 3.50 & 3.44 \\
\hline 16 & Lab. Object Oriented Programming & D.S & 3.59 & 3.47 & 3.47 & 3.47 & 3.47 & 3.53 & 3.59 & 3.35 & 3.47 & 3.35 \\
\hline 17 & Web Programming & D.S & 3.26 & 3.09 & 2.91 & 3.13 & 3.00 & 3.30 & 3.48 & 3.17 & 2.96 & 3.22 \\
\hline 18 & Lab. Web Programming & D.S & 3.21 & 3.00 & 3.05 & 3.00 & 3.16 & 3.16 & 3.32 & 3.16 & 3.16 & 3.21 \\
\hline 19 & Network Security & D.S & 3.10 & 3.06 & 2.77 & 2.87 & 2.94 & 3.03 & 3.03 & 2.90 & 3.00 & 3.03 \\
\hline 20 & IT Budgetting & Ed & 3.50 & 3.50 & 3.50 & 3.50 & 3.50 & 3.50 & 3.75 & 3.50 & 3.50 & 3.50 \\
\hline 21 & English Language 1 & H.M & 3.70 & 3.65 & 3.70 & 3.65 & 3.65 & 3.65 & 3.48 & 3.61 & 3.65 & 3.57 \\
\hline 22 & Data warehouse \& Data Mining & H.W & 3.29 & 3.14 & 3.14 & 3.29 & 3.00 & 3.14 & 3.14 & 3.00 & 3.00 & 3.29 \\
\hline 23 & Web Programming & Hen & 2.94 & 2.78 & 2.67 & 2.67 & 2.61 & 2.72 & 2.89 & 2.83 & 2.72 & 2.83 \\
\hline 24 & Manajemen Information Systems & K.W & 3.11 & 2.67 & 2.94 & 2.72 & 2.72 & 2.86 & 3.11 & 2.86 & 2.89 & 2.78 \\
\hline 25 & Programming & P.Y.C & 3.18 & 3.09 & 2.77 & 2.86 & 2.77 & 3.09 & 3.14 & 3.18 & 2.91 & 3.09 \\
\hline 26 & Lab. Programming & P.Y.C & 3.39 & 3.22 & 3.05 & 3.07 & 2.93 & 3.46 & 3.39 & 3.37 & 3.15 & 3.29 \\
\hline 27 & Research Information Technology & R.A & 3.49 & 3.56 & 3.49 & 3.44 & 3.44 & 3.56 & 3.56 & 3.56 & 3.49 & 3.51 \\
\hline 28 & Management Information Systems & R.A & 3.71 & 3.79 & 3.71 & 3.71 & 3.58 & 3.67 & 3.71 & 3.79 & 3.63 & 3.75 \\
\hline 29 & Leadership & R.A & 3.60 & 3.60 & 3.40 & 3.40 & 3.40 & 3.40 & 3.40 & 3.40 & 3.40 & 3.40 \\
\hline 30 & Mandarin Language & R.A & 3.61 & 3.61 & 3.43 & 3.61 & 3.70 & 3.57 & 3.52 & 3.70 & 3.61 & 3.74 \\
\hline 31 & Business Process and Informations & Rin & 3.15 & 3.38 & 3.08 & 3.08 & 3.31 & 3.46 & 3.23 & 3.23 & 3.31 & 3.31 \\
\hline 32 & Human Computer Interaction & Rin & 3.50 & 3.10 & 3.10 & 3.30 & 3.20 & 3.30 & 3.40 & 3.40 & 3.40 & 3.40 \\
\hline 33 & Business Introduction & Rin & 3.47 & 3.47 & 3.20 & 3.27 & 3.27 & 3.33 & 3.33 & 3.33 & 3.33 & 3.40 \\
\hline 34 & English Language 2 & R.P & 3.17 & 3.02 & 2.98 & 3.00 & 3.26 & 3.19 & 2.94 & 3.07 & 3.09 & 3.07 \\
\hline 35 & English Language 4 & R.P & 3.26 & 3.45 & 3.32 & 3.42 & 3.42 & 3.39 & 3.19 & 3.55 & 3.39 & 3.45 \\
\hline 36 & Business Process and Informations & Rik & 3.89 & 3.89 & 3.89 & 3.89 & 3.89 & 3.89 & 3.89 & 3.89 & 3.89 & 3.89 \\
\hline 37 & E-Commerce & Rik & 3.20 & 3.00 & 2.80 & 3.40 & 3.00 & 3.20 & 3.00 & 3.20 & 3.20 & 3.00 \\
\hline 38 & Leadership & S.W & 3.43 & 3.38 & 3.43 & 3.29 & 3.29 & 3.38 & 3.38 & 3.24 & 3.52 & 3.19 \\
\hline 39 & Project Management & S.K & 3.04 & 2.91 & 2.88 & 2.91 & 2.91 & 2.96 & 3.00 & 2.93 & 2.89 & 2.89 \\
\hline 40 & TroubleShooting & Sud & 3.75 & 4.00 & 3.50 & 3.50 & 3.75 & 4.00 & 4.00 & 4.00 & 3.75 & 3.75 \\
\hline 41 & Web Programming & S.A & 3.40 & 3.20 & 3.00 & 3.00 & 3.20 & 2.80 & 3.20 & 2.60 & 2.80 & 3.00 \\
\hline 42 & Lab. Programming & S.A & 3.47 & 3.20 & 3.33 & 3.20 & 3.40 & 3.20 & 3.27 & 3.27 & 3.20 & 3.27 \\
\hline 43 & Web Programming & S.A.P & 3.18 & 3.18 & 3.24 & 3.41 & 3.00 & 3.06 & 3.29 & 3.06 & 3.29 & 3.06 \\
\hline 44 & Lab. Web Programming & S.A.P & 3.39 & 3.34 & 3.22 & 3.44 & 3.28 & 3.33 & 3.33 & 3.33 & 3.39 & 3.33 \\
\hline 45 & System Information Audit & T.S & 2.92 & 3.00 & 2.88 & 3.04 & 2.92 & 2.92 & 3.00 & 3.00 & 2.96 & 3.04 \\
\hline 46 & Data Structure & T.S & 2.68 & 2.68 & 2.77 & 2.82 & 2.73 & 2.77 & 2.86 & 2.68 & 2.77 & 2.64 \\
\hline 47 & Network Security & Y.C.G & 3.44 & 3.26 & 3.48 & 3.37 & 3.52 & 3.44 & 3.52 & 3.26 & 3.41 & 3.44 \\
\hline
\end{tabular}

Table VII. The Weighted Criteria

\begin{tabular}{||c|c|c|c|c|c|c|c|c|c|}
\hline E1 & E2 & E3 & E4 & E5 & E6 & E7 & E8 & E9 & E10 \\
\hline 0.0872 & 0.1086 & 0.0962 & 0.0895 & 0.0758 & 0.1552 & 0.0316 & 0.0801 & 0.1416 & 0.1342 \\
\hline \hline
\end{tabular}

Calculate the normalized decision matrix for benefit criteria:

$\mathrm{r}_{\mathrm{ij}}=\frac{\text { aij }}{\text { Max aij }} \quad \mathrm{i}=1, \ldots .47 \quad \mathrm{j}=1, \ldots .10$ 
and for cost criteria:

$\mathrm{r}_{\mathrm{ij}}=\frac{\text { Min aij }}{\text { aij }} \quad \mathrm{i}=1, \ldots .47 \quad \mathrm{j}=1, \ldots .10$

In this case of study, benefit criteria is E1, E2, E3, E5, E6, E7, E9, and E10, others is Cost criteria. The result as shown in Table VIII.

Table VIII. The Normalize Decision Matrix

\begin{tabular}{|c|c|c|c|c|c|c|c|c|c|c|}
\hline \multirow[t]{2}{*}{ Alternative } & \multicolumn{10}{|c|}{ Criteria } \\
\hline & E1 & $\overline{\mathrm{E} 2}$ & E3 & E4 & E5 & E6 & E7 & E8 & E9 & E10 \\
\hline A.B & 0.8252 & 0.7150 & 0.7352 & 0.8503 & 0.8021 & 0.7500 & 0.7850 & 0.8280 & 0.7352 & 0.7712 \\
\hline A.L & 0.8817 & 0.9100 & 0.9460 & 0.8042 & 0.8817 & 0.8750 & 0.8925 & 0.7345 & 0.9100 & 0.9177 \\
\hline $\mathrm{Alb}$ & 0.8458 & 0.7025 & 0.7866 & 0.8990 & 0.7635 & 0.7425 & 0.7650 & 0.8966 & 0.7558 & 0.7635 \\
\hline A.H & 0.8098 & 0.7500 & 0.7918 & 0.8900 & 0.7506 & 0.7700 & 0.7700 & 0.9123 & 0.7712 & 0.7712 \\
\hline A.P & 0.9692 & 0.9625 & 0.9100 & 0.7376 & 0.9692 & 0.9050 & 0.8650 & 0.7046 & 0.9306 & 0.9897 \\
\hline A.P & 0.8997 & 0.8750 & 0.8997 & 0.7417 & 0.8740 & 0.8500 & 0.8000 & 0.7429 & 0.8483 & 0.8740 \\
\hline And & 0.7121 & 0.7200 & 0.7712 & 0.9144 & 0.7404 & 0.7300 & 0.7400 & 0.8784 & 0.7815 & 0.7506 \\
\hline And & 0.7995 & 0.7775 & 0.7429 & 0.9239 & 0.7712 & 0.8050 & 0.8050 & 0.8360 & 0.7712 & 0.7995 \\
\hline Ams & 0.9203 & 0.9050 & 0.9100 & 0.7542 & 0.9203 & 0.8950 & 0.8950 & 0.7345 & 0.9203 & 0.8997 \\
\hline B.D & 0.8021 & 0.7500 & 0.7301 & 0.8669 & 0.7712 & 0.7900 & 0.8100 & 0.8228 & 0.7815 & 0.8021 \\
\hline D.W & 0.7224 & 0.7500 & 0.7609 & 0.8476 & 0.7892 & 0.7775 & 0.8150 & 0.8360 & 0.7892 & 0.7995 \\
\hline D.L & 0.8355 & 0.7975 & 0.9152 & 0.9271 & 0.8355 & 0.8275 & 0.8600 & 0.8307 & 0.8046 & 0.8689 \\
\hline D.L & 0.9075 & 0.8500 & 0.8920 & 0.7695 & 0.8226 & 0.8500 & 0.8325 & 0.7808 & 0.8920 & 0.8740 \\
\hline D.L & 0.8843 & 0.8825 & 0.8920 & 0.7899 & 0.8843 & 0.8750 & 0.8750 & 0.7855 & 0.8920 & 0.8997 \\
\hline D.S & 0.9152 & 0.8600 & 0.8997 & 0.7762 & 0.9152 & 0.8750 & 0.9075 & 0.7692 & 0.8997 & 0.8843 \\
\hline D.S & 0.9229 & 0.8675 & 0.8920 & 0.7695 & 0.8920 & 0.8825 & 0.8975 & 0.7761 & 0.8920 & 0.8612 \\
\hline D.S & 0.8380 & 0.7725 & 0.7481 & 0.8530 & 0.7712 & 0.8250 & 0.8700 & 0.8202 & 0.7609 & 0.8278 \\
\hline D.S & 0.8252 & 0.7500 & 0.7841 & 0.8900 & 0.8123 & 0.7900 & 0.8300 & 0.8228 & 0.8123 & 0.8252 \\
\hline D.S & 0.7969 & 0.7650 & 0.7121 & 0.9303 & 0.7558 & 0.7575 & 0.7575 & 0.8966 & 0.7712 & 0.7789 \\
\hline Ed & 0.8997 & 0.8750 & 0.8997 & 0.7629 & 0.8997 & 0.8750 & 0.9375 & 0.7429 & 0.8997 & 0.8997 \\
\hline H.M & 0.9512 & 0.9125 & 0.9512 & 0.7315 & 0.9383 & 0.9125 & 0.8700 & 0.7202 & 0.9383 & 0.9177 \\
\hline H.W & 0.8458 & 0.7850 & 0.8072 & 0.8116 & 0.7712 & 0.7850 & 0.7850 & 0.8667 & 0.7712 & 0.8458 \\
\hline Hen & 0.7558 & 0.6950 & 0.6864 & 1.0000 & 0.6710 & 0.6800 & 0.7225 & 0.9187 & 0.6992 & 0.7275 \\
\hline K.W & 0.7995 & 0.6675 & 0.7558 & 0.9816 & 0.6992 & 0.7150 & 0.7775 & 0.9091 & 0.7429 & 0.7147 \\
\hline P.Y.C & 0.8175 & 0.7725 & 0.7121 & 0.9336 & 0.7121 & 0.7725 & 0.7850 & 0.8176 & 0.7481 & 0.7943 \\
\hline P.Y.C & 0.8715 & 0.8050 & 0.7841 & 0.8697 & 0.7532 & 0.8650 & 0.8475 & 0.7715 & 0.8098 & 0.8458 \\
\hline R.A & 0.8972 & 0.8900 & 0.8972 & 0.7762 & 0.8843 & 0.8900 & 0.8900 & 0.7303 & 0.8972 & 0.9023 \\
\hline R.A & 0.9537 & 0.9475 & 0.9537 & 0.7197 & 0.9203 & 0.9175 & 0.9275 & 0.6860 & 0.9332 & 0.9640 \\
\hline R.A & 0.9254 & 0.9000 & 0.8740 & 0.7853 & 0.8740 & 0.8500 & 0.8500 & 0.7647 & 0.8740 & 0.8740 \\
\hline R.A & 0.9280 & 0.9025 & 0.8817 & 0.7396 & 0.9512 & 0.8925 & 0.8800 & 0.7027 & 0.9280 & 0.9614 \\
\hline Rin & 0.8098 & 0.8450 & 0.7918 & 0.8669 & 0.8509 & 0.8650 & 0.8075 & 0.8050 & 0.8509 & 0.8509 \\
\hline Rin & 0.8997 & 0.7750 & 0.7969 & 0.8091 & 0.8226 & 0.8250 & 0.8500 & 0.7647 & 0.8740 & 0.8740 \\
\hline Rin & 0.8920 & 0.8675 & 0.8226 & 0.8165 & 0.8406 & 0.8325 & 0.8325 & 0.7808 & 0.8560 & 0.8740 \\
\hline R.P & 0.8149 & 0.7550 & 0.7661 & 0.8900 & 0.8380 & 0.7975 & 0.7350 & 0.8469 & 0.7943 & 0.7892 \\
\hline R.P & 0.8380 & 0.8625 & 0.8535 & 0.7807 & 0.8792 & 0.8475 & 0.7975 & 0.7324 & 0.8715 & 0.8869 \\
\hline Rik & 1.0000 & 0.9725 & 1.0000 & 0.6864 & 1.0000 & 0.9725 & 0.9725 & 0.6684 & 1.0000 & 1.0000 \\
\hline Rik & 0.8226 & 0.7500 & 0.7198 & 0.7853 & 0.7712 & 0.8000 & 0.7500 & 0.8125 & 0.8226 & 0.7712 \\
\hline S.W & 0.8817 & 0.8450 & 0.8817 & 0.8116 & 0.8458 & 0.8450 & 0.8450 & 0.8025 & 0.9049 & 0.8201 \\
\hline S.K & 0.7815 & 0.7275 & 0.7404 & 0.9175 & 0.7481 & 0.7400 & 0.7500 & 0.8874 & 0.7429 & 0.7429 \\
\hline Sud & 0.9640 & 1.0000 & 0.8997 & 0.7629 & 0.9640 & 1.0000 & 1.0000 & 0.6500 & 0.9640 & 0.9640 \\
\hline S.A & 0.8740 & 0.8000 & 0.7712 & 0.8900 & 0.8226 & 0.7000 & 0.8000 & 1.0000 & 0.7198 & 0.7712 \\
\hline S.A & 0.8920 & 0.8000 & 0.8560 & 0.8344 & 0.8740 & 0.8000 & 0.8175 & 0.7951 & 0.8226 & 0.8406 \\
\hline S.A.P & 0.8175 & 0.7950 & 0.8329 & 0.7830 & 0.7712 & 0.7650 & 0.8225 & 0.8497 & 0.8458 & 0.7866 \\
\hline S.A.P & 0.8715 & 0.8348 & 0.8278 & 0.7762 & 0.8432 & 0.8325 & 0.8325 & 0.7808 & 0.8715 & 0.8560 \\
\hline T.S & 0.7506 & 0.7500 & 0.7404 & 0.8783 & 0.7506 & 0.7300 & 0.7500 & 0.8667 & 0.7609 & 0.7815 \\
\hline T.S & 0.6889 & 0.6700 & 0.7121 & 0.9468 & 0.7018 & 0.6925 & 0.7150 & 0.9701 & 0.7121 & 0.6787 \\
\hline Y.C.G & 0.8843 & 0.8150 & 0.8946 & 0.7923 & 0.9049 & 0.8600 & 0.8800 & 0.7975 & 0.8766 & 0.8843 \\
\hline
\end{tabular}

The Simple Additive Weighting method evaluate each alternative Preference Value (Vi).

$\mathrm{Vi}=\sum_{j=1}^{n} \mathrm{Wj} * r i j \quad \mathrm{i}=1, \ldots .47, \quad \mathrm{j}=1, \ldots .10$

By calculate the preference value from normalize decision matrix with weighted criteria we get the result, shown in Table IX and fig. 4 
Tabel IX. The Ranked Personnel

\begin{tabular}{|c|c|c|c|c|}
\hline No & Courses & Alternative & Preference Value & Rank \\
\hline 1 & Database Management System & A.B & 0.772367149 & 42 \\
\hline 2 & Entrepreneurship and Motivation & A.L & 0.880388589 & 8 \\
\hline 3 & Database Management System & Alb & 0.784752267 & 38 \\
\hline 4 & Human Computer Interaction & A.H & 0.79439309 & 34 \\
\hline 5 & English Language 2 & A.P & 0.904876924 & 3 \\
\hline 6 & English Language 4 & A.P & 0.846790828 & 18 \\
\hline 7 & Strategic Information System & And & 0.770865894 & 44 \\
\hline 8 & E-Business & And & 0.800598742 & 32 \\
\hline 9 & TroubleShooting & Ams & 0.880419684 & 7 \\
\hline 10 & Mandarin Language & B.D & 0.790070552 & 35 \\
\hline 11 & Management Information Systems & D.W & 0.785753548 & 37 \\
\hline 12 & Entrepreneurship and Motivation & D.L & 0.846485667 & 19 \\
\hline 13 & Management & D.L & 0.852848933 & 16 \\
\hline 14 & Entrepreneurship and Motivation & D.L & 0.869922029 & 11 \\
\hline 15 & Object Oriented Programming & D.S & 0.870761638 & 9 \\
\hline 16 & Lab. Object Oriented Programming & D.S & 0.866355291 & 13 \\
\hline 17 & Web Programming & D.S & 0.803801954 & 30 \\
\hline 18 & Lab. Programming Web & D.S & 0.810573056 & 27 \\
\hline 19 & Network Security & D.S & 0.788672798 & 36 \\
\hline 20 & IT Budgetting & Ed & 0.869591039 & 12 \\
\hline 21 & English Language 1 & H.M & 0.892958831 & 5 \\
\hline 22 & Data warehouse \& Data Mining & H.W & 0.806506749 & 28 \\
\hline 23 & Web Programming & Hen & 0.746368039 & 46 \\
\hline 24 & Manajemen Information Systems & K.W & 0.765228996 & 45 \\
\hline 25 & Programming & P.Y.C & 0.78392675 & 40 \\
\hline 26 & Lab. Programming & P.Y.C & 0.824765074 & 26 \\
\hline 27 & Research Information Technology & R.A & 0.870575398 & 10 \\
\hline 28 & Management Information Systems & R.A & 0.90014334 & 4 \\
\hline 29 & Leadership & R.A & 0.86014931 & 14 \\
\hline 30 & Mandarin Language & R.A & 0.885095426 & 6 \\
\hline 31 & Business Process and Informations & Rin & 0.839551631 & 22 \\
\hline 32 & Human Computer Interaction & Rin & 0.831266282 & 24 \\
\hline 33 & Business Introduction & Rin & 0.844491179 & 20 \\
\hline 34 & English Language 2 & R.P & 0.803153056 & 31 \\
\hline 35 & English Language 4 & R.P & 0.843181283 & 21 \\
\hline 36 & Business Process and Informations & Rik & 0.937244366 & 1 \\
\hline 37 & E-Commerce & Rik & 0.784088955 & 39 \\
\hline 38 & Leadership & S.W & 0.850528115 & 17 \\
\hline 39 & Project Management & S.K & 0.771724306 & 43 \\
\hline 40 & TroubleShooting & Sud & 0.925303696 & 2 \\
\hline 41 & Web Programming & S.A & 0.798734949 & 33 \\
\hline 42 & Lab. Programming & S.A & 0.830919898 & 25 \\
\hline 43 & Web Programming & S.A.P & 0.804385338 & 29 \\
\hline 44 & Lab. Programming Web & S.A.P & 0.835988413 & 23 \\
\hline 45 & System Information Audit & T.S & 0.772673422 & 41 \\
\hline 46 & Data Structure & T.S & 0.738962579 & 47 \\
\hline 47 & Network Security & Y.C.G & 0.859148512 & 15 \\
\hline
\end{tabular}

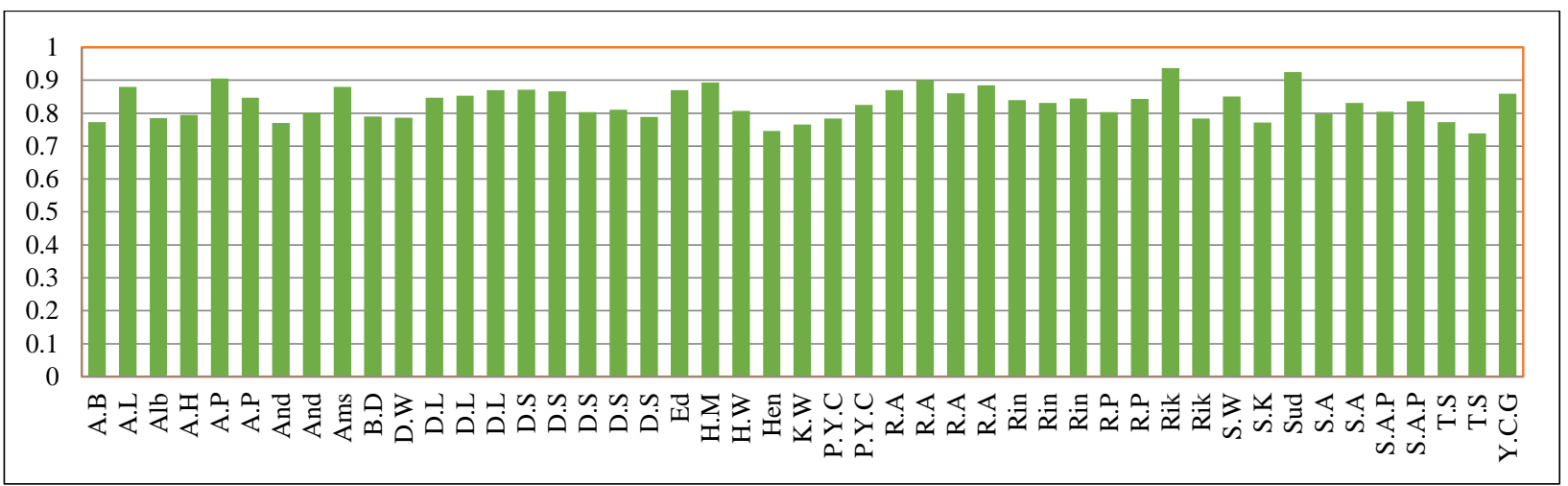

Fig.4 Lecturer Performance Evaluation Chart 


\section{CONCLUSIONS}

Multi Criteria Decision Making for evaluation of lecturer performance using the AHP and SAW methods can provide a level of consistency $90.39 \%$ from 10 indicators of criteria. With 28 lecturers for alternative, who taught 47 subjects, we rank from 28 lecturers and get the best it's Rik, followed by Sud, A.P, R.A, H.M and the others. The result of these, will be more effective and efficient in giving an assessment to management. So the management can make a decision. In this study we use data questionnaire from Faculty of Science and Technology, Information Systems Department at Buddhi Dharma University.

\section{REFERENCES}

[1] Departemen Pendidikan Nasional, "Undang undang No. 14 tahun 2005 Tentang Guru dan Dosen, Depdiknas Jakarta”, 2005.

[2] D, Sundani, "Perangkat Lunak SPSS Sebagai Alat Untuk Analisa Hubungan Kerja Dosen Dengan Keberhasilan Belajar", Jurnal Informatika, vol.13, 2008.

[3] B. Daniawan, "Evauasi Hasil Kinerja Pengajaran Dosen Menggunakan Metode TOPSIS: Studi Kasus Prodi Sistem Informasi Fakultas Sains dan Teknologi, Universitas Buddhi Dharma Tangerang", Akselerator, vol. 2 no. 2, hal. 11-18, Des. 2017.

[4] M.T. Joshua, A.M. Joshua. and W.A. Kritsonis, "Use of Student Achievement Scores as Basis for Assessing Teacher's Instructional Effectiveness", National forum of teacher education journal, vol. 17, no. 3, pp. 1-13, 2006.

[5] T.L, Saaty, "The Analytic Hierarchy Process" McGraw-Hill, New York, 1980.

[6] A. Afshari, M. Mojahed and R.M. Yusuf, "Simple Additive Weighting approach to Personnel Selection problem", International Journal of Innovation, Management and Technology, vol. 1, no. 5, December 2010 .

[7] D. Dalalah, F. Al-Oqla, and M. Hayajneh, "Application of the Analytic Hierarchy Process (AHP) in MultiCriteria Analysis of the Selection of Crane", Jordan Journal of Mechanical and Industrial Engineering, vol 4, no. 5, pp. 567 - 578, Nov. 2010.

[8] S. Nursari and V. Sciorra, "Decision Support System for Final Assignment with Analytical Hierarchy Process (AHP) Method. Case Study: Informatics Engineering Faculty of Engineering, Pancasila University", bt, vol. 1, no. 1, pp. 19-25, Sep. 2018.

[9] Marimin, "Sistem Pendukung Pengambilan Keputusan dan Sistem Pakar" IPB Press, Bogor, 2017.

[10] Abidin, "Pemilihan Strategi Hijau (Green Strategy) Pengembangan Agroindustri (Studi kasus: Agroindustri Berbasis Kelapa)", Akselerator, vol. 3 no. 1, hal. 1-13, Jun. 2018

[11] M.J. Asgharpour, "Multiple Criteria Decision Making”, University of Tehran press-Iran, 2008, pp.232, 6th Edition.

[12] A. Yani and L.D. Bakti "Competence Making on Computer Engineering Program by Using Analytical Hierarchy Process (AHP)", (IJACSA) International Journal of Advanced Computer Science and Applications, vol. 6, no. 9, 2015.

[13] T.L, Saaty, and G.V. Luis, 'Fundamentals of Decision Making and Priority Theory with the Analytic Hierarchy Process'. The Analytic Hierarchy Process, Vol. 6, 2000. 